\title{
Hausdorff and Fractal Dimension Estimates for Invariant Sets of Non-Injective Maps
}

\author{
V. A. Boichenko, A. Franz, G. A. Leonov and V. Reitmann
}

\begin{abstract}
In this paper we are concerned with upper bounds for the Hausdorff and fractal dimensions of negatively invariant sets of maps on Riemannian manifolds. We consider a special class of non-injective maps, for which we introduce a factor describing the "degree of non-injectivity". This factor can be included in the Hausdorff dimension estimates of DouadyOesterlé type $[2,7,10]$ and in fractal dimension estimates $[5,13,15]$ in order to weaken the condition to the singular values of the tangent map. In a number of cases we get better upper dimension estimates.
\end{abstract}

Keywords: Hausdorff dimension estimates, fractal dimension estimates, non-injective maps, tangent map, singular values

AMS subject classification: Primary 58 F 12, secondary 58 F 08

\section{Introduction}

In [2] Hausdorff dimension estimates for compact sets $K \subset \mathbb{R}^{n}$ that are invariant under $C^{1}$-maps $\varphi$ are given. The main idea consists in showing that for a number $j \in \mathbb{N}$ the Hausdorff outer measure of $\varphi^{j}(K)$ is by a certain factor smaller than the outer measure of $K$, i.e. the iterated map is contracting with respect to the Hausdorff outer measure on $K$. The contraction constant can be estimated by means of a singular-value function of the tangent map, i.e. if the singular-value function is less than 1 , then the map is contracting. In $[7,8]$ the condition for the contraction of the Hausdorff outer measure in $\mathbb{R}^{n}$ is weakened using Lyapunov-type functions. The latter results are generalized in [10] to maps on Riemannian manifolds (see also [6]). Using a technique similar to that of Douady and Oesterlé, Temam gave in [13] (see also [14]) upper bounds for the Hausdorff and fractal dimensions of semiflow invariant sets in a Hilbert space. Analogously fractal dimension estimates are derived in [5] for semiflows on Riemannian manifolds.

In practice the maps describing concrete physical or technical systems are often noninjective (see, for instance, [1]). For such non-injective maps it may be possible to use

V. A. Boichenko: St. Petersburg State Univ., Fac. Math. and Mech., Bibliotechnaya pl. 2, Petershof, 198904 St. Petersburg, Russia; supported by the Russ. Res. Fund 96-01-01172.

G. A. Leonov: St. Petersburg State Univ., Fac. Math. and Mech., Bibliotechnaya pl. 2, Petershof, 198904 St. Petersburg, Russia

A. Franz: Techn. Univ., Inst. Anal., Mommsenstr. 13, D - 01062 Dresden; supported by the DFG-Schwerpunktprogramm "Dynamik: Analysis, effiziente Simulation und Ergodentheorie". V. Reitmann: Techn. Univ., Inst. Anal., Mommsenstr. 13, D - 01062 Dresden

ISSN 0232-2064 / S 2.50 C Heldermann Verlag Berlin 
information about the "degree of non-injectivity" in order to get Hausdorff and fractal outer measure and dimension estimates under weakened conditions compared with the theorems mentioned above. For the first time such Douady-Oesterlé-type Hausdorff dimension estimates using the "degree of non-injectivity" are considered in [9]. There a class of $k-1$-endomorphisms is described, where the given invariant set can be split into $k$ compact subsets and where each of those subsets is mapped onto the whole invariant set. The factor $\frac{1}{k}$ can be used to compensate the missing contraction property for the Hausdorff outer measure.

In the present paper we consider a class of maps satisfying even a weaker noninjectivity condition than the $k-1$-property. In general, such a class may be described as follows. Let $\varphi$ be a $C^{1}$-map on a smooth (for simplicity $C^{\infty}$ ) $n$-dimensional Riemannian manifold $(M, g)$ and $K \subset M$ a compact set. (A class of maps that are only piecewise $C^{1}$ is considered in [11]. For these maps many results of this paper are also true.) Suppose that for a given outer measure $m(\cdot, d)$ on $M$ (d-dimensional Hausdorff or fractal outer measure of the given set or of a covering class of this set) there exist a number $0<a<1$ and a family $\left\{K_{j}\right\}_{j \geq j_{0}}$ of subsets of $K$ such that $m\left(\varphi^{j}\left(K_{j}\right), d\right)=m\left(\varphi^{j}(K), d\right)$ and $m\left(K_{j}, d\right) \leq a^{j} m(K, d)$ for all $j \geq j_{0}$. A map $\varphi$ with such properties can be considered as piecewise $m(\cdot, d)$-expansive on $K\left(\frac{1}{a}\right.$ is the expansion parameter and also describes the "degree of non-injectivity"). It follows that for such a map and any set $A \subset K$ there exists a $j \geq j_{0}$ such that $m\left(K_{j}, d\right) \leq a^{j} m(K, d) \leq m(A, d)$ and $m\left(\varphi^{j}\left(K_{j}\right), d\right)=m\left(\varphi^{j}(K), d\right)$, i.e. the semidynamical system $\left\{\varphi^{j}\right\}_{j \geq 0}$ generated by a piecewise $m(\cdot, d)$-expansive map has a certain transitive Markov-type property on $K$. It will be shown that for negatively invariant sets $K$ of piecewise $m(\cdot, d)$-expansive maps, where $m(\cdot, d)$ is the $d$-dimensional Hausdorff or fractal outer measure, the parameter $d$ is an upper bound of the associated dimension.

\section{Hausdorff dimension estimates}

First let us recall some notations of the Hausdorff outer measure and Hausdorff dimension for compact subsets $K$ of a metric space $(X, \varrho)$. Let $\varepsilon>0$ and $d \geq 0$ be arbitrary real numbers. For a fixed cover $\left\{B_{r_{i}}\right\}_{i \in I}$ of $K$ by a finite number of balls of radii $r_{i}$ the value $\sum_{i \in I} r_{i}^{d}$ is the $d$-dimensional Hausdorff outer measure of the cover. Considering all possible finite covers of $K$ by balls of radii at most $\varepsilon$ we get the $d$-dimensional Hausdorff outer measure for the covering class of $K$ by balls of radii at most $\varepsilon$ by

$$
\mu_{H}(K, d, \varepsilon)=\inf \sum_{i} r_{i}^{d}
$$

For fixed $d$ and $K$ the $d$-dimensional Hausdorff outer measure of $K$ is the limit of the monotone decreasing function $\mu_{H}(K, d, \cdot)$

$$
\mu_{H}(K, d)=\lim _{\varepsilon \rightarrow 0+0} \mu_{H}(K, d, \varepsilon) .
$$

For every compact set $K$ the uniquely defined critical number $d^{*} \geq 0$ with

$$
\mu_{H}(K, d)= \begin{cases}\infty & \text { for } 0 \leq d<d^{*} \\ 0 & \text { for } d>d^{*}\end{cases}
$$


is the Hausdorff dimension of $K$ and is denoted by $\operatorname{dim}_{H}(K)$.

To describe how a ball in an Euclidean space is transformed under a linear map, the singular values of this map are used. Let $E$ and $E^{\prime}$ be two $n$-dimensional Euclidean spaces with the scalar products $\langle\cdot, \cdot\rangle_{E}$ and $\langle\cdot, \cdot\rangle_{E^{\prime}}$, respectively, and let $L: E \rightarrow E^{\prime}$ be a lirear map. By $L^{*}$ we denote the adjoint operator, i.e. the unique linear operator $L^{*}: E^{\prime} \rightarrow E$ satisfying $\left\langle L^{*} u, v\right\rangle_{E}=\langle u, L v\rangle_{E^{\prime}}$ for all $u \in E^{\prime}$ and $v \in E$. The singular values $\alpha_{1}(L) \geq \ldots \geq \alpha_{n}(L)$ of $L$ are defined to be the eigenvalues of the positive semidefinite operator $\sqrt{L^{*} L}$. They are ordered with respect to their size and algebraic multiplicity. For an arbitrary integer $k \in\{0,1, \ldots, n\}$ we define

$$
\omega_{k}(L)= \begin{cases}\alpha_{1}(L) \cdots \alpha_{k}(L) & \text { for } k>0 \\ 1 & \text { for } k=0 .\end{cases}
$$

Furthermore, for an arbitrary number $d \in(0, n]$, further on written in the form $d=d_{0}+s$ with $d_{0} \in\{0, \ldots, n-1\}$ and $s \in(0,1]$, we define a singular-value function by

$$
\omega_{d}(L)=\omega_{d_{0}}^{1-s}(L) \omega_{d_{0}+1}^{s}(L) .
$$

For two linear maps $L: E \rightarrow E^{\prime}$ and $L^{\prime}: E^{\prime} \rightarrow E^{\prime \prime}$ between $n$-dimensional Euclidean spaces there holds the relation (see $[6,10]$ )

$$
\omega_{d}\left(L L^{\prime}\right) \leq \omega_{d}\left(L^{\prime}\right) \omega_{d}(L) \quad \text { for } d \in[0, n] .
$$

Remark 1. If we restrict ourselves to the case $\alpha_{i}(L)>0 \quad(i=1, \ldots, n)$, then the geometrical interpretation of the singular values is as follows. Let $u_{1}, \ldots, u_{n}$ be an orthonormal basis of $E$ such that $u_{i}$ is an eigenvector of $\sqrt{L^{*} L}$ corresponding to the eigenvalue $\alpha_{i}(L) \quad(i=1, \ldots, n)$. Then there exists an orthonormal basis $v_{1}, \ldots, v_{n}$ in $E^{\prime}$ such that $v_{i}=\frac{1}{\alpha_{i}(L)} L u_{i}$ for any $i=1, \ldots, n$. The image of the unit ball

$$
B_{1}(0)=\left\{a_{1} u_{1}+\ldots+a_{n} u_{n} \in E \mid\left(a_{1}, \ldots, a_{n}\right) \in \mathbb{R}^{n}, a_{1}^{2}+\ldots+a_{n}^{2} \leq 1\right\}
$$

in $E$ under the map $L$ is the set

$$
\left\{\sum_{1 \leq i \leq n} b_{i} v_{i} \in E^{\prime} \mid\left(b_{1}, \ldots, b_{n}\right) \in \mathbb{R}^{n}, \sum_{1 \leq i \leq n}\left(\frac{b_{i}}{\alpha_{i}(L)}\right)^{2} \leq 1\right\},
$$

i.e. an ellipsoid in $E^{\prime}$ where the lengths of the semiaxes are $\alpha_{1}(L), \ldots, \alpha_{n}(L)$, respectively.

Therefore a similar concept for ellipsoids is introduced. Let $\mathcal{E}$ be an ellipsoid in an $n$-dimensional Euclidean space $E$, and let $\alpha_{1}(\mathcal{E}) \geq \ldots \geq \alpha_{n}(\mathcal{E}) \geq 0$ denote the lengths of its semiaxes (not necessarily positive). This means there exists an orthonormal basis $u_{1}, \ldots, u_{n}$ in $E$ such that

$$
\mathcal{E}=\left\{\sum_{1 \leq i \leq n, \alpha_{i}(\mathcal{E}) \neq 0} a_{i} u_{i} \in E \mid a_{i} \in \mathbb{R}, \sum_{1 \leq i \leq n, \alpha_{i}(\mathcal{E}) \neq 0}\left(\frac{a_{i}}{\alpha_{i}(\mathcal{E})}\right)^{2} \leq 1\right\}
$$


For an arbitrary integer $k \in\{0,1, \ldots, n\}$ we define

$$
\omega_{k}(\mathcal{E})= \begin{cases}\alpha_{1}(\mathcal{E}) \cdots \alpha_{k}(\mathcal{E}) & \text { for } k>0 \\ 1 & \text { for } k=0\end{cases}
$$

and for an arbitrary number $d \in(0, n], d=d_{0}+s$ with $d_{0} \in\{0, \ldots, n-1\}$ and $s \in(0,1]$ we denote the $d$-dimensional ellipsoid volume by

$$
\omega_{d}(\mathcal{E})=\omega_{d_{0}}^{1-s}(\mathcal{E}) \omega_{d_{0}+1}^{s}(\mathcal{E})
$$

Now let $(M, g)$ be a smooth $n$-dimensional Riemannian manifold, $U \subset M$ an open set, $\varphi: U \rightarrow M$ a $C^{1}$-map and let $d_{u} \varphi$ denote the tangent map of $\varphi$ at the point $u \in U$. Then for an arbitrary set $K \subset U$ and a number $d \in[0, n]$ we define

$$
\omega_{d, K}(\varphi)=\sup _{u \in K}\left(\omega_{d}\left(d_{u} \varphi\right)\right)
$$

which is an upper bound for the growth rate of the $d$-dimensional ellipsoid volume of small balls in the tangent bundle over $K$ under the tangent map.

The following theorem describes the asymptotic behaviour of the $d$-dimensional Hausdorff outer measure of a compact set under a piecewise $\mu_{H}(\cdot, d)$-expansive $C^{1}$-map.

Theorem 1. Let $(M, g)$ be a smooth n-dimensional Riemannian manifold, $U \subset M$ be an open set and $\varphi: U \rightarrow M$ be a $C^{1}$-map. Suppose $K$ and $\widetilde{K}$ are compact sets satisfying the relations $K \subset \widetilde{K} \subset U$ and $\varphi^{j}(K) \subset \widetilde{K}$ for any $j \in \mathbb{N}$. Suppose that for some numbers $d \in(0, n]$ and $a>0$ the following conditions are satisfied:

1) $\omega_{d, \widetilde{K}}(\varphi)<\frac{1}{a}$.

2) There is a number $j_{0} \in \mathbb{N}$ such that for any natural number $j \geq j_{0}$ there exists a set $K_{j} \subset K$ such that $\mu_{H}\left(\varphi^{j}\left(K_{j}\right), d\right)=\mu_{H}\left(\varphi^{j}(K), d\right)$ and $\mu_{H}\left(K_{j}, d\right) \leq a^{j} \mu_{H}(K, d)$.

3) $\mu_{H}(K, d)<\infty$.

Then $\lim _{j \rightarrow \infty} \mu_{H}\left(\varphi^{j}(K), d\right)=0$.

Proof. It follows from (1) that the singular value function satisfies the relation

$$
\omega_{d, K}\left(\varphi^{j}\right) \leq \omega_{d, \widetilde{K}}^{j}(\varphi)
$$

for any $j \in \mathbb{N}$. Further, for any number $\delta>0$ using condition 1) we find a number $j_{\delta}>j_{0}$ so that for $d$ written as $d=d_{0}+s$ with $d_{0} \in\{0, \ldots, n-1\}$ and $s \in(0,1]$ the inequality

$$
2^{d_{0}}\left(d_{0}+1\right)^{\frac{d}{2}}\left(\omega_{d, \widetilde{K}}(\varphi) \cdot a\right)^{j} \leq \delta
$$

will be true for any $j>j_{\delta}$. Using additionally condition 2) and [10: Lemma 2.3] we get for any $j>j_{\delta}$ the relations

$$
\begin{aligned}
\mu_{H}\left(\varphi^{j}(K), d\right) & =\mu_{H}\left(\varphi^{j}\left(K_{j}\right), d\right) \\
& \leq 2^{d_{0}}\left(d_{0}+1\right)^{\frac{d}{2}} \omega_{d, \widetilde{K}}^{j}(\varphi) \mu_{H}\left(K_{j}, d\right) \\
& \leq 2^{d_{0}}\left(d_{0}+1\right)^{\frac{d}{2}}\left(\omega_{d, \widetilde{K}}(\varphi) \cdot a\right)^{j} \mu_{H}(K, d) \\
& \leq \delta \mu_{H}(K, d) .
\end{aligned}
$$

Since $\delta$ can be chosen arbitrarily small, by condition 3 ) we get $\lim _{j \rightarrow \infty} \mu_{H}\left(\varphi^{j}(K), d\right)=$ 0 
Corollary 1. If the conditions 1) and 2) of Theorem 1 are satisfied for certain numbers $a>0$ and $d \in(0, n]$ and furthermore $\varphi(K) \supset K$ holds, then either $\mu_{H}(K, d)=$ 0 , or $\mu_{H}(K, d)=\infty$.

Corollary 2. Let the conditions 2) and 3) of Theorem 1 be satisfied for certain numbers $a>0$ and $d \in(0, n]$. Furthermore, let $p: \widetilde{K} \rightarrow \mathbb{R}_{+}=\{x \in \mathbb{R} \mid x>0\}$ be a continuous function such that the condition

$$
\sup _{u \in \widetilde{K}}\left(\frac{p(\varphi(u))}{p(u)} \omega_{d}\left(d_{u} \varphi\right)\right)<\frac{1}{a}
$$

is satisfied. Then $\lim _{j \rightarrow \infty} \mu_{H}\left(\varphi^{j}(K), d\right)=0$.

Proof. The proof is based on a technique considering $p$ as Lyapunov-type function, similarly to [8] for $M=\mathbb{R}^{n}$. It follows from condition (4) that there is a number $0<\nu<1$ with $a \frac{p(\varphi(u))}{p(u)} \omega_{d}\left(d_{u} \varphi\right)<\nu$ for any $u \in \widetilde{K}$. Therefore by the chain rule and by applying (1) we get for any $u \in \widetilde{K}$ and arbitrary $j \in \mathbb{N}$

$$
\begin{aligned}
a^{j} \omega_{d}\left(d_{u} \varphi^{j}\right) & \leq a^{j} \omega_{d}\left(d_{\varphi^{j-1}(u)} \varphi\right) \cdots \omega_{d}\left(d_{u} \varphi\right) \\
& \leq a^{j} \frac{\nu}{a} \frac{p\left(\varphi^{j-1}(u)\right)}{p\left(\varphi^{j}(u)\right)} \cdots \frac{\nu}{a} \frac{p(u)}{p(\varphi(u))} \\
& =\nu^{j} \frac{p(u)}{p\left(\varphi^{j}(u)\right)} \\
& \leq \nu^{j} \frac{\sup _{u \in \widetilde{K}^{j}} p(u)}{\inf _{u \in \widetilde{K}} p(u)} .
\end{aligned}
$$

For any $\delta>0$ we find a number $j_{\delta}>j_{0}$ such that for $d\left(d=d_{0}+s\right.$ with $d_{0} \in$ $\{0, \ldots, n-1\}$ and $s \in(0,1])$ the relation

$$
2^{d_{0}}\left(d_{0}+1\right)^{\frac{d}{2}} a^{j} \omega_{d, \widetilde{K}^{\prime}}\left(\varphi^{j}\right) \leq \delta
$$

will be true for any $j>j_{\delta}$. For these numbers $j$ we get, similarly as in the proof of Theorem 1,

$$
\begin{aligned}
\mu_{H}\left(\varphi^{j}(K), d\right) & =\mu_{H}\left(\varphi^{j}\left(K_{j}\right), d\right) \\
& \leq 2^{d_{0}}\left(d_{0}+1\right)^{\frac{d}{2}} \omega_{d, \widetilde{K}}\left(\varphi^{j}\right) \mu_{H}\left(K_{j}, d\right) \\
& \leq 2^{d_{0}}\left(d_{0}+1\right)^{\frac{d}{2}} \omega_{d, \widetilde{K}}\left(\varphi^{j}\right) a^{j} \mu_{H}(K, d) \\
& \leq \delta \mu_{H}(K, d)
\end{aligned}
$$

and therefore, $\lim _{j \rightarrow \infty} \mu_{H}\left(\varphi^{j}(K), d\right)=0$

Example 1. Consider a class of modified horseshoe maps $\varphi$ defined on the unit $n$-cube $Q=[0,1] \times \ldots \times[0,1] \subset \mathbb{R}^{n}$. The map $\varphi$ contracts $Q$ with a factor $\alpha<\frac{1}{2}$ in the first $(n-1)$ coordinate directions $x_{1}, \ldots, x_{n-1}$ and stretches it with the factor $\beta_{1}>1$ if $x_{n}<h$ and with the factor $\beta_{2}>\beta_{1}$ if $x_{n}>h(0<h<1)$ in the remaining direction $x_{n}$. Then the resulting parallelepiped is folded along the hyperplane $H=$ 
$\left\{\left(x_{1}, \ldots, x_{n}\right) \in \mathbb{R}^{n} \mid x_{n}=h\right\}$ seperating the regions with different stretching factors, and finally it is formed to an $n$-dimensional horseshoe. Figure 1 illustrates such a map in the two-dimensional case.

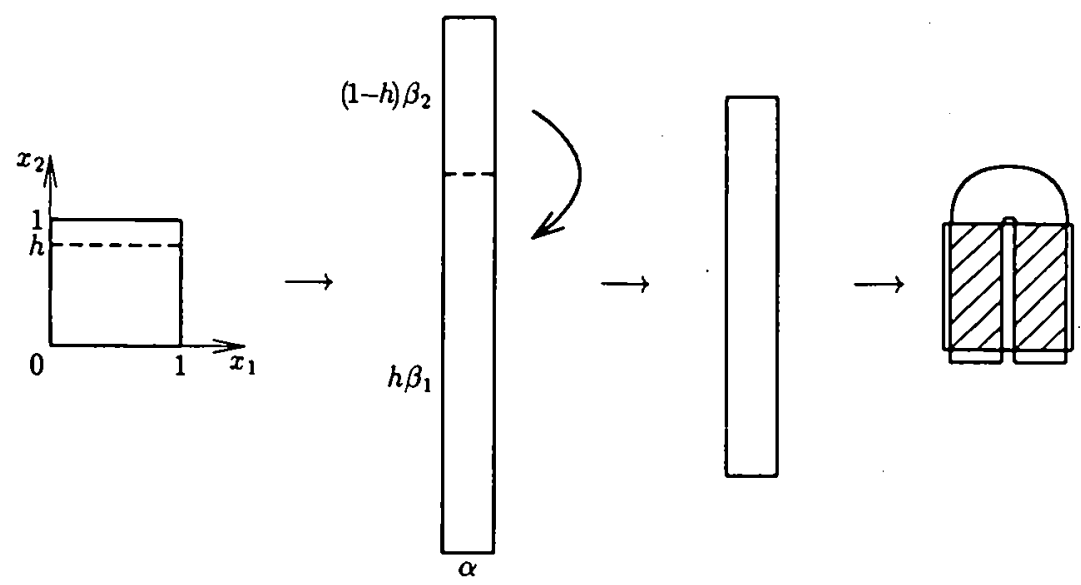

Figure 1: Modified horseshoe map

Further we assume that the map can be extended continuously differentiable to an open neighbourhood $U$ of $K=\bigcap_{i=-\infty}^{\infty} \varphi^{i}(Q)$, where $\varphi^{i}(\cdot)$ for negative numbers $i$ means the preimage under the map $\varphi^{-i}$. Let us consider the case $\alpha^{n-1} \beta_{2} \geq 1$, where the Theorem of Douady and Oesterlé (see [2]) is not applicable.

The set $K$ is invariant under the map. For $\widetilde{K}=K$ the condition $\varphi^{j}(K) \subset \widetilde{K}$ is satisfied for each $j \in \mathbb{N}$. Choose $K_{1}$ as that part of $K$ where the stretching factor of $\varphi$ is $\beta_{1}$. Then $\varphi\left(K_{1}\right)=K$. Iteratively define $K_{j}=K_{j-1} \cap \varphi^{-1}\left(K_{j-1}\right)(j \geq 2)$. By induction there is $\varphi\left(K_{j}\right)=K_{j-1}$ and therefore, $\varphi^{j}\left(K_{j}\right)=K=\varphi^{j}(K)$. That means the first part of condition 2) of Theorem 1 holds. Furthermore, in the two-dimensional case illustrated in Figure 1 it can be shown that $K_{j}$ consists of $4^{j}$ linear copies of $K^{\prime}$ obtained by horizontal contraction with factor $\alpha^{j}$ and vertical contraction with factor $\left(\frac{1}{\beta_{1}}\right)^{j}$. The $4^{j}$ parts of $K_{j}$ are disjoint and compact, and therefore have a certain distance $2 \varepsilon_{j}>0$. In a cover of $K$ by balls of radius smaller than $\varepsilon_{j}$ every ball can contain points of only one of the $4^{j}$ parts of $K_{j}$. Therefore, taking $\varepsilon<\varepsilon_{j}$ and $d \in[0,2]$ we have

$$
\mu_{H}\left(K_{j}, d, \varepsilon\right)=\left(4 \gamma^{d}\right)^{j} \mu_{H}\left(K, d, \gamma^{-j} \varepsilon\right),
$$

where $\gamma=\min \left\{\alpha, \frac{1}{\beta_{1}}\right\}$. Passing to the limit for $\varepsilon \rightarrow 0+0$ we get the second part of condition 2) of Theorem 1 with $a=4 \gamma^{d}$.

It remains to check condition 1). Because of $\alpha \beta_{2} \geq 1$ we can restrict ourselves to $d \in[1,2]$. Now the singular-value function has the form $\omega_{d, K}(\varphi)=\beta_{2} \alpha^{d-1}$, thus we are looking for a number $d \in[1,2]$ satisfying

$$
4 \gamma^{d} \beta_{2} \alpha^{d-1}<1
$$


This is equivalent to $d>\frac{\ln \alpha-\ln 4 \beta_{2}}{\ln \alpha+\ln \gamma}$. For instance, for the parameters $\alpha=\frac{1}{3}, \beta_{1}=3$, $\beta_{2}=5$ we get $d>\frac{1}{2}\left(\frac{\ln 20}{\ln 3}+1\right) \approx 1.863$. For such numbers $d$ the conditions 1$)$ and 2 ) of Theorem 1 are satisfied, and Corollary 1 yields $\mu_{H}(K, d)=0$ or $\mu_{H}(K, d)=\infty$

Now we want to use the same method as above to find an upper estimate of the Hausdorff dimension of the set $K$ considered in Theorem 1. Let us additionally assume that $K$ is negatively invariant under $\varphi$, i.e. $K \subset \varphi(K)$ as in Corollary 1 . In order to find an upper bound for the Hausdorff dimension of $K$ we can not assume $\mu_{H}(K, d)<\infty$ as in Theorem 1. However it is possible to consider the Hausdorff outer measure of the class of finite covers of $K$ by balls of radii at most $\varepsilon$ instead of the Hausdorff outer measure of $K$ itself, because the outer measure of a finite cover is always finite.

Theorem 2. Let $(M, g)$ be a smooth $n$-dimensional Riemannian manifold, $U \subset M$ be an open set and $\varphi: U \rightarrow M$ be a $C^{1}$-map. Suppose $K$ and $\widetilde{K}$ are compact sets satisfying the relation $K \subset \varphi^{j}(K) \subset \widetilde{K} \subset U$ for any $j \in \mathbb{N}$. Suppose that for some numbers $a>0$ and $d \in(0, n]$ of the form $d=d_{0}+s$ with $d_{0} \in\{0, \ldots, n-1\}$ and $s \in(0,1]$ the following conditions are satisfied:

1) $\omega_{d, \widetilde{k}}(\varphi)<\frac{1}{a}$.

2) There are numbers $l$ with $\omega_{d, \widetilde{K}}(\varphi)<l<\frac{1}{a}$ and $j_{0} \in \mathbb{N}$ such that for any natural number $j>j_{0}$ there exist a set $K_{j} \subset K$ and a number $\varepsilon_{j}>0$ such that

$$
\begin{aligned}
\mu_{H}\left(\varphi^{j}\left(K_{j}\right), d, \varepsilon\right) & =\mu_{H}\left(\varphi^{j}(K), d, \varepsilon\right) \\
\mu_{H}\left(K_{j}, d,\left(d_{0}+1\right)^{-\frac{1}{2}} l^{-\frac{j}{d}} \varepsilon\right) & \leq a^{j} \mu_{H}(K, d, \varepsilon)
\end{aligned}
$$

holds for any $\varepsilon \in\left(0, \varepsilon_{j}\right]$.

Then $\operatorname{dim}_{H}\left(\Pi^{-}\right) \leq d$.

Proof. Like in the proof of Theorem 1 we have $\omega_{d, K}\left(\varphi^{j}\right) \leq \omega_{d, \widetilde{K}^{j}}(\varphi)<l^{j}$ for any $j \in \mathbb{N}$. For any $\delta>0$ we find an integer $j_{\delta}>j_{0}$ so that the relation $2^{d_{0}}\left(d_{0}+1\right)^{\frac{d}{2}}(a l)^{j}<\delta$ will be true for any $j>j_{\delta}$. Now let $j>j_{\delta}$ be fixed and consider $0<\varepsilon \leq \min \left\{\varepsilon_{j},\left(d_{0}+\right.\right.$ 1.) $\left.)^{-\frac{1}{2}} l^{-\frac{j}{d}} \varepsilon_{0}\left(l^{j}\right)\right\}$, where $\varepsilon_{0}$ is defined in [10: Lemma 2.3]. Then condition 2) and [10: Lemma 2.3 ] result in the following inequalities:

$$
\begin{aligned}
\mu_{H}(K, d, \varepsilon) & \leq \mu_{H}\left(\varphi^{j}\left(K^{*}\right), d, \varepsilon\right) \\
& =\mu_{H}\left(\varphi^{j}\left(K_{j}\right), d, \varepsilon\right) \\
& \leq 2^{d_{0}}\left(d_{0}+1\right)^{\frac{d}{2}} l^{j} \mu_{H}\left(K_{j}, d,\left(d_{0}+1\right)^{-\frac{1}{2}} l^{-\frac{j}{d}} \varepsilon\right) \\
& \leq 2^{d_{0}}\left(d_{0}+1\right)^{\frac{d}{2}}(a l)^{j} \mu_{H}(K, d, \varepsilon) \\
& \leq \delta \mu_{H}(K, d, \varepsilon) .
\end{aligned}
$$

Since the number $\delta$ can be choosen arbitrarily small and $\mu_{H}(K, d, \varepsilon)$ is finite, this means $\mu_{H}\left(K^{j}, d, \varepsilon\right)=0$ for any $\varepsilon \in\left(0, \min \left\{\varepsilon_{j},\left(d_{0}+1\right)^{\left.\left.-\frac{1}{2} l^{-\frac{i}{d}} \varepsilon_{0}\left(l^{j}\right)\right\}\right)}\right.\right.$ and therefore, $\mu_{H}\left(K^{-}, d\right)=0$. Hence we get $\operatorname{dim}_{H}\left(K^{*}\right) \leq d$ 
Using now a Lyapunov-type function we get a corollary of this theorem analogous to Corollary 2.

Corollary 3. Let $(M, g), U, K, \widetilde{K}$ and $\varphi$ be defined as in Theorem 2, and let $p: \widetilde{K} \rightarrow \mathbb{R}_{+}$be a continuous function, such that for some numbers $a>0$ and $d \in(0, n]$, $d=d_{0}+s$ with $d_{0} \in\{0, \ldots, n-1\}$ and $s \in(0,1]$ the following conditions are satisfied:

1) $\sup _{u \in \widetilde{K}}\left(\frac{p(\varphi(u))}{p(u)} \omega_{d}\left(d_{u} \varphi\right)\right)<\frac{1}{a}$.

2) There are numbers $l$ with $\sup _{u \in \widetilde{K}}\left(\frac{p(\varphi(u))}{p(u)} \omega_{d}\left(d_{u} \varphi\right)\right)<l<\frac{1}{a}$ and $j_{0} \in \mathbb{N}$ such that for any natural number $j>j_{0}$ there exist a set $K_{j} \subset K$ and a number $\varepsilon_{j}>0$ with

$$
\begin{aligned}
& \mu_{H}\left(\varphi^{j}\left(K_{j}\right), d, \varepsilon\right)=\mu_{H}\left(\varphi^{j}(K), d, \varepsilon\right)
\end{aligned}
$$

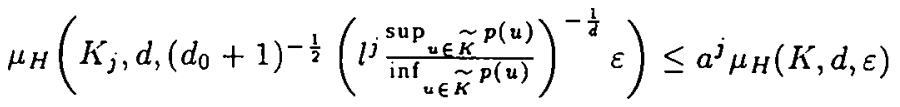

for any $\varepsilon \in\left(0, \varepsilon_{j}\right]$.

Then $\operatorname{dim}_{H}(K) \leq d$.

Example 2 (Example 1 continued). For the modified horseshoe map described before, in the two-dimensional case the first part of condition 2) of Theorem 2 is satisfied for arbitrary numbers $\varepsilon>0$ and $d \in[1,2]$. Furthermore, we can show the existence of a number $l$ with $\omega_{d, K}(\varphi)<l<\frac{1}{a}$ satisfying $\left(d_{0}+1\right)^{-\frac{1}{2}} l^{-\frac{j}{d}} \geq \gamma^{j}$. Together with (5) this yields the second part of condition 2). Thus we get $\operatorname{dim}_{H}(K) \leq d$ for any number $d>\frac{\ln \alpha-\ln 4 \beta_{2}}{\ln \alpha+\ln \gamma}$. In the limit this yields $\operatorname{dim}_{H}(K) \leq \frac{\ln \alpha-\ln 4 \beta_{2}}{\ln \alpha+\ln \gamma}$. For the parameters $\alpha=\frac{1}{3}, \beta_{1}=3$ and $\beta_{2}=5$ we get $\operatorname{dim}_{H}(K) \leq 1.863$.

If $\varphi\left(K \backslash K_{1}\right) \subset K_{1}$ holds, then the dimension estimate can be improved by means of Corollary 3. Using an appropriate Lyapunov-type function the condition 1) of Theorem 2 can be replaced by condition 1 ) of Corollary 3 . Since here the singular-value function is constant on $K_{1}$ and $K \backslash K_{1}$, respectively, the simplest type of Lyapunov function is of the same kind, i.e. $p(u)=1$ for $u \in K_{1}$ and $p(u)=P>0$ for $u \in K \backslash K_{1}$. Since the distance between the sets $K_{1}$ and $K^{\prime} \backslash K_{1}$ is positive, such a function is continuous on $\widetilde{K}=K$. The constant $P$ has to be chosen in such a way that $\sup _{u \in K}\left(\frac{p(\varphi(u))}{p(u)} \omega_{d}\left(d_{u} \varphi\right)\right)$ becomes minimal. Because of

$$
\frac{p(\varphi(u))}{p(u)} \omega_{d}\left(d_{u} \varphi\right)= \begin{cases}\frac{1}{P} \beta_{2} \alpha^{d-1} & \text { for } u \in K \backslash K_{1} \\ P \beta_{1} \alpha^{d-1} & \text { for } u \in K_{1}, \varphi(u) \in K \backslash K_{1} \\ \beta_{1} \alpha^{d-1} & \text { for } u \in K_{1}, \varphi(u) \in K_{1}\end{cases}
$$

we have to choose $P$ such that $\frac{1}{P} \beta_{2}=P \beta_{1}$, i.e. $P=\sqrt{\beta_{2} / \beta_{1}}$. Thus we get the Lyapunov-type function

$$
p(u)= \begin{cases}1 & \text { for } u \in K_{1} \\ \sqrt{\beta_{2} / \beta_{1}} & \text { for } u \in K \backslash K_{1}\end{cases}
$$


With this function $p$ for $d \in[1,2]$ we get

$$
\sup _{u \in K}\left(\frac{p(\varphi(u))}{p(u)} \omega_{d}\left(d_{u} \varphi\right)\right)=\sqrt{\beta_{1} \beta_{2}} \alpha^{d-1}
$$

which is less than $\omega_{d, K}(\varphi)=\beta_{2} \alpha^{d-1}$. For any number $d>\frac{\ln \alpha-\ln 4 \sqrt{\beta_{1} \beta_{2}}}{\ln \alpha+\ln \gamma}$ the conditions of Corollary 3 are satisfied, and we get the improved estimate $\operatorname{dim} H(K) \leq \frac{\ln \alpha-\ln 4 \sqrt{\beta_{1} \beta_{2}}}{\ln \alpha+\ln \gamma}$. For the parameters $\alpha=\frac{1}{3}, \beta_{1}=3$ and $\beta_{2}=5$ this means $\operatorname{dim}_{H}(K) \leq 1.747$

Remark 2. In Example 2 we would have got the same improved result if we had changed the standard metric on $\mathbb{R}^{2}$ by multiplying the metric tensor with the Lyapunovtype function $p$.

Since condition 2) of Theorem 2 is not easy to check, especially if the map is not piecewise linear, we now give some stronger conditions which can be checked more easily.

Corollary 4. Let $(M, g)$ be a smooth $n$-dimensional Riemannian manifold, $U \subset M$ an open set, $\varphi: U \rightarrow M$ a $C^{1}$-map and $K \subset U$ a compact $\varphi$-invariant set. Suppose that for some numbers $a>0$ and $d \in(0, n]$ of the form $d=d_{0}+s$ with $d_{0} \in\{0, \ldots, n-1\}$ and $s \in(0,1]$ the following conditions are satisfied:

1) $\omega_{d, k}(\varphi)<\frac{1}{a}$.

2) There is a number $j_{0} \in \mathbb{N}$ such that for any natural number $j \geq j_{0}$ there exist a set $K, \subset K$ with $\varphi^{j}\left(K_{j}\right)=K$, a natural number $N_{j}$, a number $l_{0}$ and $C^{1}$-maps $f_{i, j}: U \rightarrow M\left(i=1, \ldots, N_{j}\right)$ with

$$
K_{j}=\bigcup_{i=1}^{N_{j}} f_{i, j}\left(K^{j}\right), \quad \max _{i=1, \ldots, N_{j}} \omega_{d, K}\left(f_{i, j}\right)<l_{0}^{j}, \quad N_{j} \leq 2^{-d_{0}}\left(d_{0}+1\right)^{-\frac{d}{2}} a^{j} l_{0}^{-j}
$$

Then $\operatorname{dim} H(\Pi) \leq d$.

Proof. Using [10: Lemma 2.3], for any $j \in \mathbb{N}$ with $j>j_{0}$ there exists a number $\varepsilon_{j}$ such that

$$
\begin{aligned}
\mu_{H}\left(K_{j}, d, \sqrt{d_{0}+1} l_{0}^{j} \varepsilon\right) & \leq \sum_{i=1}^{N_{j}} 2^{d_{0}}\left(d_{0}+1\right)^{\frac{d}{2}} l_{0}^{j} \mu_{H}(K, d, \varepsilon) \\
& =N_{j} 2^{d_{0}}\left(d_{0}+1\right)^{\frac{d}{2}} l_{0}^{j} \mu_{H}(K, d, \varepsilon) \\
& \leq a^{j} \mu_{H}(K, d, \varepsilon)
\end{aligned}
$$

holds for any $\varepsilon \in\left(0, \varepsilon_{j}\right]$. Because of $N_{j} \geq 1$ and of condition 2$)$ we have $2^{-d_{0}}\left(d_{0}+\right.$ $1)^{-\frac{d}{2}} a^{j} l_{0}^{-j} \geq 1$ and therefore, $2^{d_{0}}\left(d_{0}+1\right)^{\frac{d}{2}} l_{0}^{j} \omega_{d, K}^{j}(\varphi)<1$ for any $j \geq j_{0}$. This means $l_{0} \omega_{d, k}(\varphi)<1$, and becausc of condition 1$)$, there are numbers $l \in \mathbb{R}$ and $j_{0} \in \mathbb{N}$ such that $\omega_{d, K}(\varphi)<l<\frac{1}{a}$ and $\left(l_{0} l\right)^{\frac{j}{d}}<\frac{1}{d_{0}+1}$ for any $j>j_{0}$ are satisfied. For these numbers $j$ and all $\varepsilon \in\left(0, \varepsilon_{j}\right)$ we have

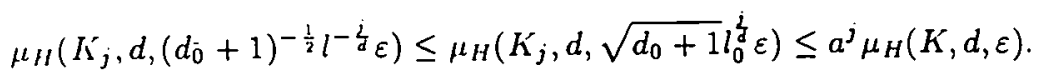

Applying Theorem 2 we get $\operatorname{dim}_{H}\left(K^{\circ}\right) \leq d$ 
Example 3 (Example 1 continued). Since in our example of the modified horseshoe map (two-dimensional) the set $K_{j}$ consists of $4^{j}$ linear copies of $K$ we define $f_{i, j}$ to be the linear map of $K$ onto the $i$ th piece of $K_{j} \quad\left(i=1, \ldots, 4^{j}\right)$. Then for $N_{j}=4^{j}$ and $a>4 l_{0}>4 \gamma^{d}$ condition 2) of Corollary 4 is satisfied. Condition 1) results in $a \beta_{2} \alpha^{d-1}<1$, and the limit for $a \rightarrow 4 \gamma^{d}$ yields (6). In this way we get the same result as before without a Lyapunov-type function, but we could reach it with less expense

\section{Fractal dimension estimates}

First let us recall the notation of the fractal outer measure and fractal dimension for compact subsets $K$ of a metric space $(X, \varrho)$. For a given number $\varepsilon>0$ let $N_{\varepsilon}(K)$ denote the smallest number of balls of radius $\varepsilon$ needed to cover $K$. Then for $d \geq 0$ the $d$-dimensional fractal outer measure of the covering class of $K$ by balls of radius $\varepsilon$ is the number

The upper limit

$$
\mu_{F}\left(K, d_{:} \varepsilon\right)=N_{\varepsilon}(K) \varepsilon^{d}
$$

$$
\mu_{F}(K, d)=\limsup _{\varepsilon \rightarrow 0+0} \mu_{F}(K, d, \varepsilon)
$$

is the $d$-dimensional fractal outer measure of $K$. The uniquely defined critical number $d^{*} \geq 0$ satisfying

$$
\mu_{F}(K, d)= \begin{cases}\infty & \text { for } 0 \leq d<d^{*} \\ 0 & \text { for } d>d^{*}\end{cases}
$$

is called the upper capacity dimension [6], upper box-counting dimension [4] or fractal dimension $[3,13-15]$ of the set $K$. It can be shown that $\operatorname{dim}_{H}(K) \leq \operatorname{dim}_{F}(K)$. In order to estimate the fractal dimension of a negatively invariant set of a $C^{1}$-map on a Riemannian manifold we need two lemmata, which are formulated and proved analogously to assertions of $[2,10]$.

Lemma 1. Let $\left(E,(\cdot,\rangle_{E}\right)$ be an $n$-dimensional Euclidean space, $u_{1}, \ldots, u_{n}$ an orthonormal basis and

$$
\mathcal{E}=\left\{a_{1} u_{1}+\ldots+a_{n} u_{n} \in E \mid\left(a_{1}, \ldots, a_{n}\right) \in \mathbb{R}^{n},\left(\frac{a_{1}}{\alpha_{1}(\mathcal{E})}\right)^{2}+\ldots+\left(\frac{a_{n}}{\alpha_{n}(\mathcal{E})}\right)^{2} \leq 1\right\}
$$

an ellipsoid with $\alpha_{1}(\mathcal{E}) \geq \ldots \geq \alpha_{n}(\mathcal{E})>0$. Then for any $\eta>0$, the set $\mathcal{E}+B_{\eta}(0)$, where $B_{\eta}(0)$ denotes the ball with radius $\eta$ centered at the origin, is contained in the ellipsoid $\mathcal{E}^{\prime}=\left(1+\frac{\eta}{\alpha_{n}(\mathcal{E})}\right) \mathcal{E}$.

Proof. We have

$$
B_{\alpha_{n}(\mathcal{E})}(0)=\left\{a_{1} u_{1}+\ldots+a_{n} u_{n} \in E \mid\left(\frac{a_{1}}{\alpha_{n}(\mathcal{E})}\right)^{2}+\ldots+\left(\frac{a_{n}}{\alpha_{n}(\mathcal{E})}\right)^{2} \leq 1\right\} \subset \mathcal{E}
$$

and therefore,

$$
\mathcal{E}+B_{\eta}(0)=\mathcal{E}+\frac{\eta}{\alpha_{n}(\mathcal{E})} B_{\alpha_{n}(\mathcal{E})}(0) \subset \mathcal{E}+\frac{\eta}{\alpha_{n}(\mathcal{E})} \mathcal{E}=\left(1+\frac{\eta}{\alpha_{n}(\mathcal{E})}\right) \mathcal{E},
$$

i.e. the statement of the lemma 
Lemma 2. Let $\left(E,(\cdot,\rangle_{E}\right)$ be an $n$-dimensional Euclidean space, $u_{1}, \ldots, u_{n}$ an orthonormal basis,

$$
\mathcal{E}=\left\{a_{1} u_{1}+\ldots+a_{n} u_{n} \in E \mid\left(\frac{a_{1}}{\alpha_{1}(\mathcal{E})}\right)^{2}+\ldots+\left(\frac{a_{n}}{\alpha_{n}(\mathcal{E})}\right)^{2} \leq 1\right\}
$$

an ellipsoid with $\alpha_{1}(\mathcal{E}) \geq \ldots \geq \alpha_{n}(\mathcal{E})>0$ and $0<r<\alpha_{n}(\mathcal{E})$. Then the relation $N_{\sqrt{n} r}(\mathcal{E}) \leq \frac{2^{n} \omega_{n}(\mathcal{E})}{r^{n}}$ holds, where $\omega_{n}(\mathcal{E})$ is defined in $(2)$.

Proof. The ellipsoid $\mathcal{E}$ is contained in the parallelepiped

$$
P=\left\{a_{1} u_{1}+\ldots+a_{n} u_{n} \in E|| a_{1}\left|\leq \alpha_{1}(\mathcal{E}), \ldots,\right| a_{n} \mid \leq \alpha_{n}(\mathcal{E})\right\}
$$

whereas the edges have the lengths $2 \alpha_{1}(\mathcal{E}), \ldots, 2 \alpha_{n}(\mathcal{E})$, respectively. This parallelepiped can be covered by

$$
\prod_{j=1}^{n}\left(\left[\frac{\alpha_{j}(\mathcal{E})}{r}\right]+1\right) \leq 2^{n} \prod_{j=1}^{n} \frac{\alpha_{j}(\mathcal{E})}{r}=\frac{2^{n} \omega_{n}(\mathcal{E})}{r^{n}}
$$

cubes with edges of the length $2 r$ parallel to the directions $u_{i}(i=1, \ldots, n)$ where [.] denotes the integer part. Each of the cubes is contained in a ball of radius $\sqrt{n} r$

The first theorem in this section provides an upper bound for the fractal dimension of a negatively invariant set if no information about the "degree of non-injectivity" is known.

Theorem 3. Let $(M, g)$ be a smooth $n$-dimensional Riemannian manifold, $U \subset M$ be an open set and $\varphi: U \rightarrow M$ be a $C^{1}$-map. Suppose $K \subset U$ is a compact set satisfying the relation $K \subset \varphi(K) \subset U$. Assume that

$$
0<\rho_{K}(\varphi):=\min _{u \in K} \alpha_{n}\left(d_{u} \varphi\right)<n^{-\frac{1}{2}}
$$

and there-cxists a number $d \in(0, n]$ such that

$$
\omega_{n, K}(\varphi) \rho_{K}^{d-n}(\varphi) \leq 8^{-n} n^{-\frac{d}{2}}
$$

Then $\operatorname{dim}_{F}(K) \leq d$.

Proof. Let $\eta \in\left(0, \rho_{k}(\varphi)\right)$ be an arbitrary number and $r_{1}>0$ be so small that there exists an open set $V \subset M$ containing $K$ which itself lies inside a compact subset of $U$ such that

$$
\left\|\tau_{\varphi(v)}^{\varphi(u)} d_{v} \varphi \tau_{u}^{v}-d_{u} \varphi\right\| \leq \eta
$$

for any $u, v \in V$ with $\varrho(u, v) \leq r_{1}$ is satisfied, where $\|\cdot\|$ here denotes the operator norm. By $\varrho(\cdot, \cdot)$ we mean the geodesic distance between the points of $M$ and by $\tau_{u}^{v}$ we denote the isometry between the tangent spaces $T_{u} M$ and $T_{v} M$ defined by parallel transport. Let $\exp _{u}: T_{u} M \rightarrow M$ denote the exponential map at an arbitrary point $u \in M$. Since $\exp _{u}$ is a smooth map satisfying $\left\|d_{O_{u}} \exp _{u}\right\|=1$ for any point $u \in M$ we 
find a number $r_{u}>0$ such that $\left\|d_{v} \exp _{u}\right\| \leq 2$ for any $v \in B_{r_{u}}\left(O_{u}\right)$, where $O_{u}$ denotes the origin of the tangent space $T_{u} M$. Since $V$ is contained in a compact set there is a number $r_{2}>0$ such that $\left\|d_{v} \exp _{u}\right\| \leq 2$ is satisfied for any $u \in V$ and any $v \in B_{r_{2}}\left(O_{u}\right)$. Furthermorc, there is a number $\alpha>0$ such that $\alpha_{1}\left(d_{u} \varphi\right)<\alpha$ is satisfied for any $u \in V$.

Now we can find a number $r_{0} \leq \min \left\{r_{1}, \frac{r_{2}}{2+\alpha+\eta}\right\}$ such that any ball $B_{r_{0}}(u)$ containing points of $K$ is entirely contained in $V$. Let $r \in\left(0, r_{0}\right)$ be fixed. Since $K$ is compact there is a finite number of points $u_{j} \in V\left(j=1, \ldots, N_{r}(K)\right)$ such that $K=\bigcup_{j=1}^{N_{r}(K)} B_{r}\left(u_{j}\right) \cap K$ and therefore,

$$
\varphi(K)=\bigcup_{j=1}^{N_{r}(K)} \varphi\left(B_{r}\left(u_{j}\right) \cap K\right)
$$

is satisfied. The Taylor formula for the differentiable map $\varphi$ guarantees the relation

$$
\begin{aligned}
& \left\|\exp _{\varphi\left(u_{j}\right)}^{-1} \varphi(v)-d_{u_{j}} \varphi\left(\exp _{u_{j}}^{-1}(v)\right)\right\| \\
& \quad \leq \sup _{w \in B_{r}\left(u_{j}\right)}\left\|\tau_{\varphi(w)}^{\varphi\left(u_{j}\right)} d_{w} \varphi \tau_{u_{j}}^{w}-d_{u_{j}} \varphi\right\| \cdot\left\|\exp _{u_{j}}^{-1}(w)\right\|
\end{aligned}
$$

for every $v \in B_{r}\left(u_{j}\right)$. Thus, using (9) and (10), the image of every ball $B_{r}\left(u_{j}\right)$ under $\varphi$ satisfies the inclusion

$$
\varphi\left(B_{r}\left(u_{j}\right)\right) \subset \exp _{\varphi\left(u_{j}\right)}\left(d_{u_{j}} \varphi\left(B_{r}\left(O_{u_{j}}\right)\right)+B_{\eta r}\left(O_{\varphi\left(u_{j}\right)}\right)\right)
$$

Since $\left.\mathcal{E}_{j}:=d_{u_{j}} \varphi\left(B_{1}\left(O_{\varphi\left(u_{j}\right)}\right)\right)\right)$ is an ellipsoid in $E=T_{\varphi\left(u_{j}\right)} M$ we get for this $\mathcal{E}_{j}$ and $\mathcal{E}_{j}^{\prime}=\left(1+\frac{\eta}{\alpha_{n}\left(\mathcal{E}_{j}\right)}\right) \mathcal{E}_{j}$ with Lemma 1

$$
\varphi\left(B_{r}\left(u_{j}\right)\right) \subset \exp _{\varphi\left(u_{j}\right)}\left(r\left(\mathcal{E}_{j}+B_{\eta}\left(O_{\varphi\left(u_{j}\right)}\right)\right)\right) \subset \exp _{\varphi\left(u_{j}\right)}\left(r \mathcal{E}_{j}^{\prime}\right)
$$

With

$$
\sigma:=\sqrt{n} \rho_{K}(\varphi)
$$

we have

$$
N_{\sigma r}\left(\varphi\left(K^{\prime}\right)\right) \leq N_{r}\left(K^{\prime}\right) \max _{j=1, \ldots, N_{r}(K)} N_{\sigma r}\left(\exp _{\varphi\left(u_{j}\right)}\left(r \mathcal{E}_{j}^{\prime}\right)\right)
$$

and therefore,

$$
\mu_{F}\left(\varphi\left(K^{\prime}\right), d, \sigma r\right) \leq\left(\sigma^{d} \max _{j=1, \ldots, N_{r}(K)} N_{\sigma r}\left(\exp _{\varphi\left(u_{j}\right)}\left(r \mathcal{E}_{j}^{\prime}\right)\right)\right) \mu_{F}(K, d, r) .
$$

Every ball $B_{\sigma r}(v)(v \in M)$ containing points of $\exp _{\varphi\left(u_{j}\right)}\left(r \mathcal{E}_{j}^{\prime}\right)$ is contained in the ball $B_{\left(2+\alpha_{1}\left(\mathcal{E}_{j}^{\prime}\right)\right) r}\left(u_{j}\right) \subset B_{r_{2}}\left(u_{j}\right)$, and so we have $B_{\sigma r}(v) \supset \exp _{\varphi\left(u_{j}\right)}\left(B_{\frac{1}{2} \sigma r}\left(\exp _{\varphi\left(u_{j}\right)}^{-1} v\right)\right)$. This
means

$$
N_{\sigma r}\left(\exp _{\varphi\left(u_{j}\right)}\left(r \mathcal{E}_{j}^{\prime}\right)\right) \leq N_{\frac{1}{2} \sigma r}\left(r \mathcal{E}_{j}^{\prime}\right)
$$

Since $\rho_{K}(\varphi) \leq \alpha_{n}\left(d_{u}, \varphi\right)=\alpha_{n}\left(\mathcal{E}_{j}\right) \leq \alpha_{n}\left(\mathcal{E}_{j}^{\prime}\right)$ is satisficd, Lemma 2 yields

$$
N_{\frac{1}{2} \sigma r}\left(r \mathcal{E}_{j}^{\prime}\right) \leq \frac{2^{n} \omega_{n}\left(r \mathcal{E}_{j}^{\prime}\right)}{\left(\frac{1}{2} r \rho_{K}(\varphi)\right)^{n}}=\frac{4^{n} \omega_{n}\left(\mathcal{E}_{j}^{\prime}\right)}{\rho_{K}^{n}(\varphi)} \leq \frac{4^{n}\left(1+\frac{\eta}{\rho_{K}(\varphi)}\right)^{n} \omega_{n}\left(\mathcal{E}_{j}\right)}{\rho_{K}^{n}(\varphi)} \leq \frac{8^{n} \omega_{n}\left(d_{u_{j}} \varphi\right)}{\rho_{K}^{n}(\varphi)}
$$


Using (11), (12) and assumption (8) we get

$$
\begin{aligned}
\mu_{F}(K, d, \sigma r) & \leq \mu_{F}(\varphi(K), d, \sigma r) \\
& \leq \sigma^{d} \frac{8^{n} \omega_{n, K}(\varphi)}{\rho_{K}^{n}(\varphi)} \mu_{F}(K, d, r) \\
& =n^{\frac{d}{2}} 8^{n} \omega_{n, K}(\varphi) \rho_{K}^{d-n}(\varphi) \mu_{F}(K, d, r) \\
& <\mu_{F}(K, d, r) .
\end{aligned}
$$

Because of (8) we have $\sigma<1$. Therefore, for any $\varepsilon \in\left(0, r_{0}\right)$ we can find a number $l \in \mathbb{N}_{0}$ such that $\sigma^{l+1} r_{0} \leq \varepsilon<\sigma^{l} r_{0}$ is satisfied. Finally we get

$$
\mu_{F}(K, d, \varepsilon)<\mu_{F}\left(K, d, \sigma^{-1} \varepsilon\right)<N_{\sigma^{-1} \varepsilon}(K) r_{0}^{d} \leq N_{\sigma r_{0}}(K) r_{0}^{d}=\sigma^{-d} \mu_{F}\left(K, d, \sigma r_{0}\right)
$$

which yields $\mu_{F}(K, d)<\infty$ and thus $\operatorname{dim}_{F}(K) \leq d$

Corollary 5. Let $(M, g), U$ and $\varphi$ be as in Theorem $3, K$ and $\widetilde{K}$ be compact sets satisfying the relation $K \subset \varphi^{j}(K) \subset \widetilde{K} \subset U$ and $\rho_{\widetilde{K}}(\varphi)>0$. Suppose that there exists a number $d \in(0, n]$ with

$$
\omega_{n, \mathbb{K}^{(}}(\varphi) \rho_{\widetilde{K}}^{d-n}(\varphi)<1
$$

Then $\operatorname{dim}_{F}(K) \leq d$.

Proof. From $K \subset \varphi^{j}(K) \subset \widetilde{K} \subset U$ we get $K \subset \varphi^{i}(K) \subset U$ for any $i \in \mathbb{N}$. With respect to (1) the iterates of $\varphi$ satisfy the relations

$$
\omega_{n, K}\left(\varphi^{i}\right) \leq \omega_{n, \widetilde{K}^{i}}^{i}(\varphi) \quad \text { and } \quad \rho_{K}\left(\varphi^{i}\right) \geq \rho_{\widetilde{K}}^{i}(\varphi)
$$

and therefore,

$$
\omega_{n, K}\left(\varphi^{i}\right) \rho_{K}^{d-n}\left(\varphi^{i}\right) \leq\left(\omega_{n, \widetilde{K}}(\varphi) \rho_{\widetilde{K}}^{d-n}(\varphi)\right)^{i} .
$$

Furthermore, we have from the definition (3)

$$
\omega_{n, K} \cdot\left(\varphi^{i}\right) \rho_{K}^{d-n}\left(\varphi^{i}\right) \geq \rho_{K}^{n}\left(\varphi^{i}\right) \rho_{K}^{d-n}\left(\varphi^{i}\right)=\rho_{K}^{d}\left(\varphi^{i}\right)
$$

By using (13) - (15), without loss of generality we can assume

$$
\omega_{n, k}(\varphi) \rho_{K}^{d-n}(\varphi) \leq 8^{-n} n^{-\frac{d}{2}} \quad \text { and } \quad \rho_{K}(\varphi)<n^{-\frac{1}{2}}
$$

In the opposite case consider the map $\varphi^{i}$ with sufficiently large $i$. With Theorem 3 wc get $\operatorname{dim}_{F}(K) \leq d$

Corollary 6. Let $(M, g), U, K$ and $\varphi$ be defined as in Theorem 3 and $p: U \rightarrow \mathbb{R}_{+}$ be a continuous function. Suppose that the following conditions are satisfied:

1) $\omega_{n}\left(d_{n} \varphi\right)=$ const $\neq 0$ for all $u \in K$.

2) There exists a number $s \in(0,1]$ such that $\frac{p(\varphi(u))}{p(u)} \omega_{n-1+s}\left(d_{u} \varphi\right)<1$. for all $u \in K$.

Then $\operatorname{dim} p(K) \leq n-1+s$. 
Proof. According to condition 2) there exists a positive number $\nu<1$ with

$$
\frac{p(\varphi(u))}{p(u)} \omega_{n-1+s}\left(d_{u} \varphi\right) \leq \nu
$$

for any $u \in K$. Thercfore, by the chain rule and by (1) we have

$$
\begin{aligned}
\omega_{n-1+s, K}\left(\varphi^{i}\right) & \leq \max _{u \in K} \frac{p(u)}{p\left(\varphi^{i}(u)\right)} \frac{p\left(\varphi^{i}(u)\right)}{p\left(\varphi^{i-1}(u)\right)} \omega_{n-1+s}\left(d_{\varphi^{i-1}(u)} \varphi\right) \ldots \frac{p(\varphi(u))}{p(u)} \omega_{n-1+s}\left(d_{u} \varphi\right) \\
& \leq \frac{\max _{u \in K} p(u)}{\min _{u \in K} p(u)} \nu^{i} .
\end{aligned}
$$

Furthermore, the relation $\omega_{n-1+s, k}\left(\varphi^{i}\right) \geq \rho_{K}^{n-1+s}\left(\varphi^{i}\right)$ holds. Therefore, without loss of generality we can assume that $\omega_{n-1+g, k}(\varphi)<8^{-n} n^{\frac{n-1+3}{2}}$ and $\rho_{K}(\varphi)<n^{-\frac{1}{2}}$ is satisfied. In the opposite case consider $\varphi^{i}$ with sufficiently large $i$. We take $u_{0} \in K$ such that $\alpha_{n}\left(d_{u_{0}} \varphi\right)=\rho_{K}(\varphi)$. Resulting from (3) and condition 1) we obtain

$$
\omega_{n, h}(\varphi) \rho_{K}^{s-1}(\varphi)=\omega_{n}\left(d_{u_{0}} \varphi\right) \alpha_{n}^{s-1}\left(d_{u_{0}} \varphi\right)=\omega_{n-1+s}\left(d_{u_{0}} \varphi\right)<8^{-n} n^{\frac{n-1+\rho}{2}} .
$$

With Theorem 3 we get $\operatorname{dim}_{F}(K) \leq n-1+s$

Remark 3. Conditions analogous to 1) of Corollary 6 are considered in [15] for invertible maps as the Hénon system. In contrast to our results the fractal dimension estimates in [15] are given in terms of Lyapunov exponents and without use of a Lyapunov-type function $p$.

Now we want to include the "degree of non-injectivity" in the method of estimating the fractal dimension developed in Theorem 3.

Theorem 4. Let $(M, g)$ be a smooth n-dimensional Ricmannian manifold, $U \subset M$ be an open set and $\varphi: U \rightarrow M$ be a $C^{1}$-map. Suppose $K \subset U$ is a compact set satisfying the relation $K \subset \varphi(K) \subset U$. Suppose $\rho_{K}(\varphi)>0$, and let $a, b>0$ be numbers such that the following conditions are satisfied:

1) There exists a number $d \in(0, n]$ with

$$
\begin{aligned}
\rho_{K}(\varphi) & <a^{-\frac{1}{n}} b^{\frac{d-n}{n} n} n^{-\frac{1}{2}} \\
\omega_{n, K}(\varphi) \rho_{K}^{d-n}(\varphi) & \leq a^{-\frac{d}{n}} b^{\frac{d}{n}(d-n)} 8^{-n} n^{-\frac{d}{2}} .
\end{aligned}
$$

2) For any $j \in \mathbb{N}$ there are a compact set $K_{j} \subset K$ and a number $\varepsilon_{j}>0$ such that.

$$
\begin{aligned}
\mu_{F}\left(\varphi^{j}\left(K_{j}\right), d, \varepsilon\right) & =\mu_{F}\left(\varphi^{j}(K), d, \varepsilon\right) \\
\mu_{F}\left(K_{j}, d, b^{j} \varepsilon\right) & \leq a^{j} \mu_{F}(K, d, \varepsilon)
\end{aligned}
$$

for any $\varepsilon \in\left(0, \varepsilon_{j}\right)$ are satisfied.

Then $\operatorname{dim}_{F}\left(\Lambda^{-}\right) \leq d$. 
Proof. Analogous to the proof of Theorem 3 let $\eta \in\left(0, \rho_{K}(\varphi)\right)$ be an arbitrary number. Let $r_{1}, r_{2}>0$ be so small that there exists an open set $V \subset M$ containing $K$ and that $V$ is a contained in a compact subset of $U$ such that the inequalities $\left\|\tau_{\varphi(v)}^{\varphi(u)} d_{v} \varphi \tau_{u}^{v}-d_{u} \varphi\right\| \leq \eta$ for any $u, v \in V$ with $\varrho(u, v) \leq r_{1}$ and $\left\|d_{v} \exp _{u}\right\| \leq 2$ for any $u \in V$ and any $v \in B_{r_{2}}\left(O_{u}\right)$ are satisfied. With $\alpha$ defined in the proof of Theorem 3 we can find a number $r_{0} \leq \min \left\{r_{1}, \frac{r_{2}}{2+\alpha+\eta}, \varepsilon_{1}\right\}$ such that any ball $B_{r_{0}}(u)$ containing points of $K$ is entirely contained in $V$. Let $r \in\left(0, r_{0}\right)$ be fixed. Since $K_{1}$ is compact, there is a finite number of points $u_{j} \in V\left(j=1, \ldots, N_{r}\left(K_{1}\right)\right)$ such that $K_{1}=\bigcup_{j=1}^{N_{r}\left(K_{1}\right)} B_{r}\left(u_{j}\right) \cap K_{1}$ and therefore,

$$
\varphi\left(K_{1}\right)=\bigcup_{j=1}^{N_{r}\left(K_{1}\right)} \varphi\left(B_{r}\left(u_{j}\right) \cap K_{1}\right)
$$

is satisfied. Using the Taylor formula we get that the image of every ball $B_{r}\left(u_{j}\right)$ under $\varphi$ satisfies the inclusion

$$
\varphi\left(B_{r}\left(u_{j}\right)\right) \subset \exp _{\varphi\left(u_{j}\right)}\left(d_{u_{j}} \varphi\left(B_{r}\left(O_{u_{j}}\right)\right)+B_{\eta r}\left(O_{\varphi\left(u_{j}\right)}\right)\right) .
$$

With $\mathcal{E}_{j}:=d_{u_{j}} \varphi\left(B_{1}\left(O_{\varphi\left(u_{j}\right)}\right)\right)$ and $\mathcal{E}_{j}^{\prime}=\left(1+\frac{\eta}{\alpha_{n}\left(\mathcal{E}_{j}\right)}\right) \mathcal{E}_{j}$ we get by means of Lemma 1

$$
\varphi\left(B_{r}\left(u_{j}\right)\right) \subset \exp _{\varphi\left(u_{j}\right)}\left(r\left(\mathcal{E}_{j}+B_{\eta}\left(O_{\varphi\left(u_{j}\right)}\right)\right)\right) \subset \exp _{\varphi\left(u_{j}\right)}\left(r \mathcal{E}_{j}^{\prime}\right) .
$$

With $\sigma:=a^{\frac{1}{n}} b^{\frac{n-d}{n}} \sqrt{n} \rho_{K}(\varphi)$ we obtain

$$
N_{\sigma r}\left(\varphi\left(K_{1}\right)\right) \leq N_{b r}\left(K_{1}\right) \max _{j=1, \ldots, N_{r}\left(K_{1}\right)} N_{\sigma r}\left(\exp _{\varphi\left(u_{j}\right)}\left(b r \mathcal{E}_{j}^{\prime}\right)\right)
$$

and therefore,

$$
\mu_{F}\left(\varphi\left(K_{1}\right), d, \sigma r\right) \leq\left(\sigma^{d} b^{-d} \max _{j=1, \ldots, N_{r}\left(K_{1}\right)} N_{\sigma r}\left(\exp _{\varphi\left(u_{j}\right)}\left(b r \mathcal{E}_{j}^{\prime}\right)\right)\right) \mu_{F}(K, d, r) .
$$

Analogous to the proof of Thcorem 3 we get $N_{\sigma r}\left(\exp _{\varphi\left(u_{j}\right)}\left(b r \mathcal{E}_{j}^{\prime}\right)\right) \leq N_{\frac{1}{2} \sigma r}\left(b r \mathcal{E}_{j}^{\prime}\right)$. Since $\rho_{K}(\varphi) \leq \alpha_{n}\left(d_{u,} \varphi\right)=\alpha_{n}\left(\mathcal{E}_{j}\right) \leq \alpha_{n}\left(\mathcal{E}_{j}^{\prime}\right)$ is satisfied, Lemma 2 yields

$$
\begin{aligned}
N_{\frac{1}{2} \sigma r}\left(b r \mathcal{E}_{j}^{\prime}\right) & \leq \frac{2^{n} \omega_{n}\left(b r \mathcal{E}_{j}^{\prime}\right)}{\left(\frac{1}{2} a^{\frac{1}{n}} b^{\frac{n-d}{n}} r \rho_{K}(\varphi)\right)^{n}}=\frac{4^{n} b^{d} \omega_{n}\left(\mathcal{E}_{j}^{\prime}\right)}{a \rho_{K}^{n}(\varphi)} \leq \frac{4^{n} b^{d}\left(1+\frac{\eta}{\rho_{K}(\varphi)}\right)^{n} \omega_{n}\left(\mathcal{E}_{j}\right)}{a \rho_{K}^{n}(\varphi)} \\
& \leq \frac{8^{n} b^{d} \omega_{n}\left(d_{u_{j}} \varphi\right)}{a \rho_{K}^{n}(\varphi)} .
\end{aligned}
$$

Thus we have

$$
\begin{aligned}
\mu_{F}(K, d, \sigma r) & \leq \mu_{F}(\varphi(K), d, \sigma r) \\
& =\mu_{F}\left(\varphi\left(K_{1}\right), d, \sigma r\right) \\
& \leq \sigma^{d} b^{-d} \frac{8^{n} b^{d} \omega_{n}\left(d_{u_{j}} \varphi\right)}{a \rho_{K}^{n}(\varphi)} \mu_{F}\left(K_{1}, d, b r\right) \\
& \leq a^{\frac{d}{n}} b^{\frac{d}{n}(n-d)} n^{\frac{d}{2}} \frac{8^{n} \omega_{n}\left(d_{u_{j}} \varphi\right)}{a \rho_{K}^{n}(\varphi)} \mu_{F}\left(K_{1}, d, b r\right) \\
& \leq \mu_{F}(K, d, r) .
\end{aligned}
$$

Since $\sigma<1$, analogous to the end of the proof of Theorem 3 , this yields $\operatorname{dim}_{F}(K) \leq d$ 
Corollary 7. Let $(M, g), U$ and $\varphi$ be as in Theorem $4, K$ and $\widetilde{K}$ be compact sets satisfying the relation $K \subset \varphi^{j}(K) \subset \widetilde{K} \subset U$ and $\rho_{\widetilde{K}}(\varphi)>0$. Let condition 2) of Theorem 4 be satisfied and assume that there exists a number $d \in(0, n]$ with

$$
\omega_{n, \widetilde{K}}(\varphi) \rho_{\widetilde{K}}^{d-n}(\varphi)<a^{-\frac{d}{n}} b^{\frac{d}{n}(d-n)} .
$$

Then $\operatorname{dim}_{F}(K) \leq d$.

Proof. From $K \subset \varphi^{j}(K) \subset \tilde{K} \subset U$ we get $K \subset \varphi^{i}(K) \subset U$ for any $i \in \mathbb{N}$. The iterates of $\varphi$ satisfy the relations

$$
\omega_{n, K}\left(\varphi^{i}\right) \leq \omega_{n, \widetilde{K}^{i}}^{i}(\varphi) \quad \text { and } \quad \rho_{K}\left(\varphi^{i}\right) \geq \rho_{\widetilde{K}}^{i}(\varphi)
$$

and therefore

$$
a^{\frac{d i}{n}} b^{\frac{d i}{n}(n-d)} \omega_{n, K}\left(\varphi^{i}\right) \rho_{K}^{d-n}\left(\varphi^{i}\right) \leq\left(a^{\frac{d}{n}} b^{\frac{d}{n}(n-d)} \omega_{n, K^{\prime}}(\varphi) \rho_{\widetilde{K}}^{d-n}(\varphi)\right)^{i} .
$$

Furthermore,

$$
a^{\frac{d}{n}} b^{\frac{d}{n}(n-d)} \omega_{n, K}\left(\varphi^{i}\right) \rho_{K}^{d-n}\left(\varphi^{i}\right) \geq a^{\frac{d}{n}} b^{\frac{d}{n}(n-d)} \rho_{K}^{n}\left(\varphi^{i}\right) \rho_{K}^{d-n}\left(\varphi^{i}\right)=\left(a^{\frac{1}{n}} b^{\frac{n-d}{n}} \rho_{K}\left(\varphi^{i}\right)\right)^{d}
$$

holds. Thus without loss of generality we can assume

$$
a^{\frac{d}{n}} b^{\frac{d}{n}(n-d)} \omega_{n, K}(\varphi) \rho_{K}^{d-n}(\varphi) \leq 8^{-n} n^{-\frac{d}{2}} \quad \text { and } \quad a^{\frac{1}{n}} b^{\frac{n-d}{n}} \rho_{K}(\varphi)<n^{-\frac{1}{2}} .
$$

Otherwise consider the map $\varphi^{i}$ with sufficiently large $i$ and substitute $a$ by $a^{i}$ and $b$ by $b^{i}$. With Theorem 4 we get $\operatorname{dim}_{F}(K) \leq d$

Example 4 (Example 1 continued). Let us again consider the modified horseshoe map in two dimensions. For the sets $K_{j}$ defined before, analogous to (5) we have $N_{e}\left(K_{j}\right) \leq 4^{j} N_{\frac{c}{\gamma^{j}}}\left(K^{j}\right)$ for sufficiently small $\varepsilon>0$, i.e. with $a=4 \gamma^{d}$ and $b=\gamma$ condition 2) of Theorem 4 is satisfied. Then condition (16) results in

$$
4^{\frac{d}{2}} \gamma^{d} \beta_{2} \alpha^{d-1}<1
$$

which is equivalent to $d>\frac{\ln \alpha-\ln \beta_{2}}{\ln 2+\ln \alpha+\ln \gamma}$. Corollary 7 can be applied for any such $d$ and shows that $\operatorname{dim} F(K) \leq \frac{\ln \alpha-\ln \beta_{2}}{\ln 2+\ln \alpha+\ln \gamma}$. For the parameters $\alpha=\frac{1}{3}, \beta_{1}=3$ and $\beta_{2}=5$ we get $\operatorname{dim}_{F}(K) \leq 1.800$.

By changing the metric with the Lyapunov-type function $p$ used in Example 2 we alter the form of the balls covering $K \backslash K_{1}$. However, again we have $N_{\gamma^{j}}\left(K_{j}\right) \leq$ $4^{j} N_{\varepsilon}(K)$, i.e. condition 2) holds with $a=4 \gamma^{d}$ and $b=\gamma$. Condition (16) now results in $4^{\frac{d}{2}} \gamma^{d} \sqrt{\beta_{1} \beta_{2}} \alpha^{d-1}<1$, which means $\operatorname{dim}_{F}(K) \leq \frac{\ln \alpha-\frac{1}{2} \ln \beta_{1} \beta_{2}}{\ln 2+\ln \alpha+\ln \gamma}$. For $\alpha=\frac{1}{3}, \beta_{1}=3$ and $\beta_{2}=5$ we $\operatorname{get} \operatorname{dim}_{F}(K) \leq 1.631$.

Remark 4. For two-dimensional horseshoe maps the upper bound for the fractal dimension of an invariant set obtained by Corollary 7 is always smaller than the bound for the Hausdorff dimension by Theorem 2, because for $d<2$ condition (17) is weaker than condition (6). For $d>2$ this relation is reversed, i.e. for the considered horseshoe maps in more than two dimensions the estimates of Section 2 really will be useful. 


\section{References}

[1] Belykh, V. N.: Models of discrete systems of phase synchronization (in Russian). In: Systems of phase synchronization (eds.: V. V. Shakhgil'dyan and L. N. Belyustina). Moscow: Radio i Svyaz' 1982.

[2] Douady, A. et J. Oesterlé: Dimension de Hausdorff des attracteurs. C.R. Acad. Sci. Paris Ser. A 290 (1980), $1135-1138$.

[3] Eden, A.: Local Lyapunov exponents and a local estimate of Hausdorff dimension. Math. Modelling and Num. Anal. 23 (1989), $405-413$.

[4] Falconer, K. J.: Fractal Geometry: Mathematical Foundations and Applications. Chichester: John Wiley \& Sons 1990.

[5] Heineken, W.: Fractal Dimension Estimates for Invariant Sets of Vector Fields. Diploma thesis. Dresden: Techn. Univ. 1997.

[6] Ledrappier, F.: Some relations between dimension and Lyapunov exponents. Commun. Math. Phys. 81 (1981), $229-283$.

[7] Leonov, G. A. and V. A. Boichenko: Lyapunov's direct method in the estimation of the Hausdorff dimension of allractors. Acta Appl. Math. 26 (1992), 1 - 60.

[8] Leonov, G. A., Burkin, I. M. and A. I. Shepelyawyi: Frequency Methods in Oscillation Theory. Dordrecht: Kluwer Acad. Publ. 1996.

[9] Mirle, A.: Hausdorff dimension estimates for invariant sets of $k-1$-maps. Preprint 25/95, DFG-Schwerpunktprogranın "Dynamik: Analysis, effiziente Simulation und Ergodentheorie" 1995.

[10] Noack, A. and V. Reitmaun: Hausdorff dimension of invariant sets of time-dependent vector fields. Z. Anal. Anw. 15 (1996), $457-473$.

[11] Reitmann, V. and U. Schnabel: Hausdorff dimension estimates for invariant sets of piecewise smooth maps. Z. Ang. Math. Mech. (ZAMM) (to appear).

[12] Simon, K.: Hausdorff dimension for non-invertible maps. Ergod. theory \& Dyn. Sys. 13 (1993), $199-212$.

[13] Temam, R.: Infinite-Dimensional Dynamical Systems in Mechanics and Physics. New York: Springer 1988.

[14] Thieullen, P.: Entropy and the Hausdorff dimension for infinite-dimensional dynamical systems. J. Dyn. Diff. Equ. 4 (1992), 127 - 159.

[15] Zhi-Min Chen: A note on Kaplan-Yorke-type estimates on the fractal dimension of chaotic attractors. Chaos, Solitons \& Fractals 3 (1993), 575 - 582. 